\title{
Persistence of regular motions for nearly-integrable Hamiltonian systems in the thermodynamic limit
}

\author{
A. Carati, L.Galgani, A. Maiocchi, F. Gangemi; R. Gangemi ${ }^{\dagger}$
}

December 24, 2016

\begin{abstract}
A review is given of the studies imed at extending to the thermodynamic limit stability results of Nekhoroshev type for nearly-integrable Hamiltonian systems. The physical relevance of such an extension, i.e., of proving the persistence of regular (or ordered) motions in that limit, is also discussed. This is made in connection both with the old Fermi, Pasta, Ulam problem, which gave origin to such discussions, and with the optical spectral lines, the existence of which was recently proven to be possible in classical models, just in virtue of such a persistence.
\end{abstract}

\section{Introduction}

Let us situate the present contribution in the frame of the scientific interests of N.N. Nekhoroshev (Kolya, for his friends), to whose memory this volume of the Journal "Regular and chaotic dynamical systems" is dedicated. Essentially, all of Kolya's works were devoted to the stability properties of nearly-integrable Hamiltonian systems (i.e., perturbations of integrable ones). Two topics were addressed by him. The first one is of a geometric character. The second topic, with which alone we will be concerned here, is of a more analytic character, and deals with the persistence of regular (or ordered) motions for nearly-integrable Hamiltonian systems.

His main general result [1] was amply illustrated by Arnol'd in his celebrated book "Supplementary chapters", and then was made popular outside of Russia by the Italian school of dynamical systems [2], that had arisen at those times between Milan, Padua and Rome. Later, he came very frequently to Milan, finally becoming a professor in that university. He used to live in the apartment of the older (LG) of the present authors, either alone, or together with Irina,

${ }^{*}$ Department of Mathematics, Università degli Studi di Milano -Via Saldini 50, I-20133 Milano, Italy. E-mail: andrea.carati@unimi.it

${ }^{\dagger}$ DMMT, Università di Brescia, Viale Europa 11, I-25123 Brescia, Italy 
sharing life with the several persons, nephews or guests or friends, that were living there in a kind of a family college. It is just from Milan that he eventually flew back to Russia for holidays, where he would shortly later be taken by the illness that led him to pass away.

The reason for the interest of the Italians to Kolya's works was a question of physics, which is related to the last work of Fermi (the famous Fermi, Pasta, Ulam, or FPU, work) [3] of the year 1954, and is indeed a deep one. By numerical computations FPU had shown that, for a discretized model of a nonlinear string, with initial data out of equilibrium (only some low-frequency modes excited), the expected equilibrium was not attained within the available integration time. Moreover, some kind of stationary state was apparently attained, with no further tendency towards the equilibrium predicted by classical statistical mechanics (energy equipartition among the normal modes). This means persistence of regular motions, against the naive expectation that, at least for systems of macroscopic size, the nonlinearity would lead to completely chaotic motions, irrespective of temperature. According to the Milan school of theoretical physics of those times (Caldirola, Loinger, Bocchieri, Scotti) the FPU result, which had been confirmed in the work [4], was a challenge for the foundations of physics. Indeed it appeared to indicate that phenomena usually explained in quantum mechanical (QM) terms (the specific heat of solids, in the FPU case) may admit of an explanation in purely classical terms (see [5], [6]). This should just be due to the persistence of regular motions that were observed in the FPU model, notwithstanding the presence of a nonlinearity. But these indications required to be confirmed or disproved on sound mathematical bases.

\section{The transition from regular to chaotic mo- tions, and its dependence on the number $n$ of degrees of freedom}

Before illustrating the way in which such a problem was later framed, we have to mention the fundamental contribution due to another Russian scientist, B.V. Chirikov. In several papers of numerical or semianalytic character (the most known of which is that written with Izrailev [7]) he pointed out that the rate of approach to equilibrium in the FPU model depends on the initial temperature (defined as the time-mean of kinetic energy, divided by the number $n$ of degrees of freedom). His result was that, if the initial temperature is sufficiently high, the "FPU paradox" disappears, because the expected equilibrium is quickly attained. So there exists a critical temperature, a threshold in temperature, above which equilibrium is quickly attained, and the paradoxical FPU phenomenon is relegated to the lower temperatures. However, there remains the problem that the threshold temperature depends on the number $n$ of degrees of freedom. So, with his great theoretical temperament, Chirikov was also able to conjecture a possible mechanism by which the "FPU paradox" would be eliminated at all from physics. His conjecture was just that, in the "thermodynamic limit" (i.e. 
in the limit $n \rightarrow \infty$ at fixed temperature), the critical or threshold temperature vanishes. In such a way, for macroscopic, large enough, systems there would be no problem at all: chaotic motions would always prevail, and no quantum-like effects would be possible in classical physics, at least in the frame of statistical mechanics, with which the FPU problem is concerned.

\section{How the problem should be reduced to an an- alytic one}

But how should one prove or disprove the Chirikov conjecture? Here one meets with a feature of scientific research which we like most: namely, determining the appropriate frame in which some problem, expressed in qualitative terms, might be suitably formulated and discussed in precise terms. Our problem is to estimate the threshold. But in which frame? The frame that was available to Chirikov was that of perturbation theory for Hamiltonian systems in the "classical sense", in which one controls all of the initial data. This is indeed the frame in which the most relevant result of a global type had been obtained by Kolya. Chirikov knew the results of the Russian school, and pointed out that, according to the estimates provided through the methods then available, the threshold would disappear in the thermodynamic limit. So his conjecture was apparently correct.

The deep reason for this result is that there exists a very special class of initial data, which in a sense are extremely improbable, for which chaotic motions occur at all temperatures. This point was for a long time debated in Italy by Benettin, Galgani and Giorgilli, who were really convinced that a way out from the Chirikov conclusion could be found, if just one were able to somehow remove those silly improbable initial data. But no solution was actually found.

\section{The statistical mechanics approach}

Eventually the way out was provided in the work [8], and indeed in an extremely simple and natural way. It suffices to take the approach of statistical mechanics, in which one has available some invariant probability measure for the initial data (typically, the Gibbs family, parametrized by temperature), and one forgets the idea of controlling each individual initial datum. One looks instead for mean values (with respect to the given measure) of some relevant dynamical variables (or observables). More precisely, one typically estimates the time correlation functions of suitable observables. Indeed, almost by definition a dynamical system is said to be chaotic if the time correlation functions of all pairs of smooth observables tend to zero for increasing time.

The key point of such an approach is that the necessity of controlling all individual initial data is eliminated, and thus one may hope that, by the proce-

dure of taking averages over all initial data, the contribution due the individual improbable initial data disappears, at least for large $n$. Another relevant feature 
of such an approach, is that relaxation times too can be defined and estimated. Taking the point of view that one should fix a priori a certain observation time (for example a time of geological size in the case of glasses) a system can be considered to be regular, with respect to a given observable, if the time autocorrelation function of that observable attains some nonvanishing value, which is apparently stationary up to the given observation time.

In the quoted paper [8], considering for simplicity sake a variant of the FPU model, known as the rotator model, stability in such a sense was proved to hold for a finite observation time for large $n$, with estimates independent of $n$, and thus also in the thermodynamic limit. For the FPU model, an analogous result was given in [9]. Such stability results are not of exponential type in the perturbation parameter. Exponential stability was then obtained for an FPU-like chain in [10] (see also [11]) and for the rotator model in [12].

\section{A quantum-like result in crystal optics, due to the persistence of regular motions: the spec- tral lines of ionic crystals in the infrared}

All problems mentioned above appear to be already very interesting both from the mathematical point of view and for their physical relevance. They are however somehow too general. One should find some concrete example having a relevant physical impact, which allows for a comparison with experimental data, and can be explained in a classical context only in virtue of the existence of regular motions. If one be allowed to compare small things to great ones, one may recall that, when QM took over, it was the comparison with experimental data (the black body spectrum with Planck, the specific heat of solids with Einstein) that played the game.

We are confident that we will be able to tackle the specific heat problem in the future. There is however another problem of a very similar type, that we already were able to tackle, in which the regular character of the motions plays a fundamental role. This is the problem of the optical spectral lines, which seems to be even more appropriate if one aims at understanding how far can one go in explaining, within classical physics, phenomena that are considered to be typical of quantum physics.

For reasons that we cannot discuss here, we are not yet able to deal with the paradigmatic case of the hydrogen lines, or more in general of lines which involve the motions of the electrons. We were able however to deal with the spectral lines of ionic crystals in the infrared, typically for the cases of Lithium Fluoride ( $\mathrm{LiF}$ ) [13] or quartz [14], which just involve the motions of the ions. It will be seen that the existence itself of the spectral lines, with their peaks, can occur in a classical context only because the motionsof the ions are of regular type. Indeed, full chaoticity would entail a flat fuzzy spectrum (see for example [15]). One might say: no regular motions, no spectral lines.

Some details are now illustrated. We consider the same model that is used 


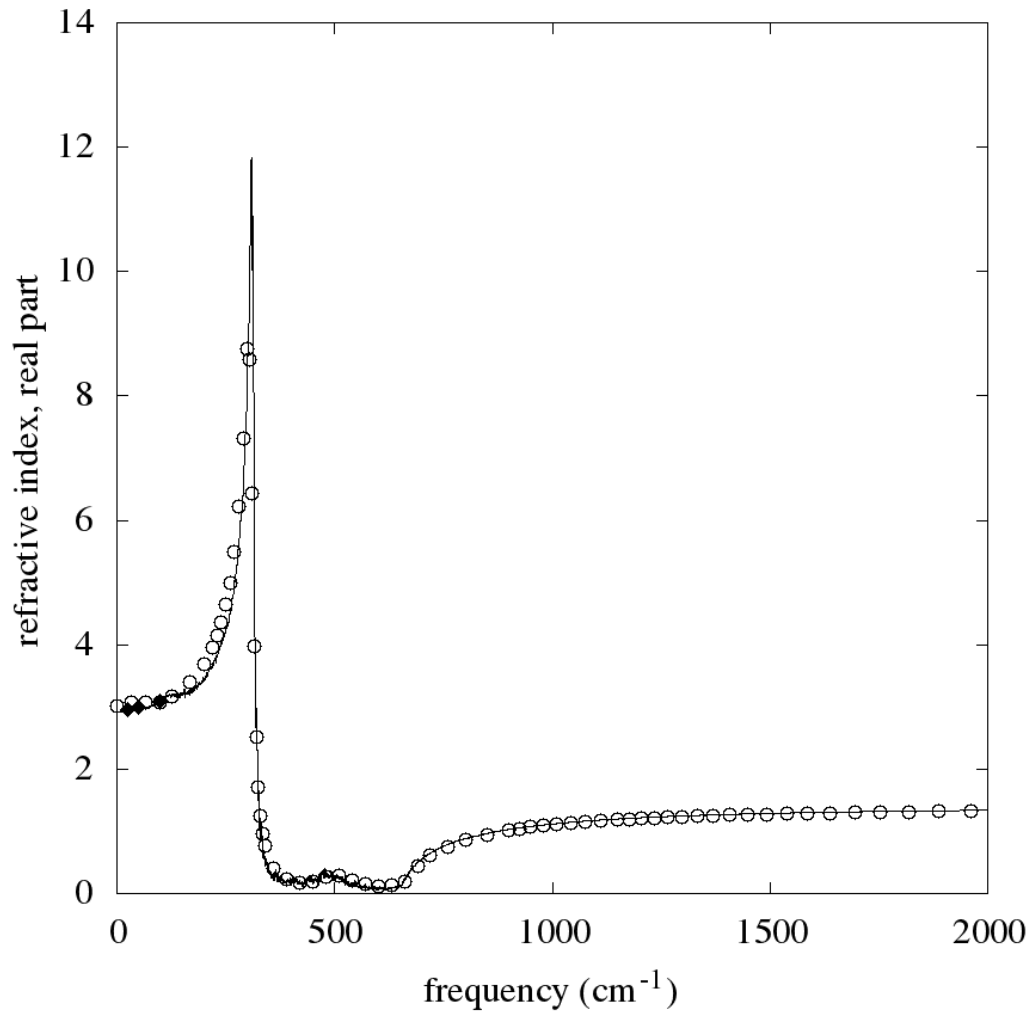

Figure 1: Real part of the refractive index $n$ vs frequency for LiF at a temperature of $300 \mathrm{~K}$. The continuous line is the theoretical curve, while the circles and the diamonds are the experimental data (see paper [13]).

in all quantum treatments. Namely, the ions are dealt with as point (rather than as extended) particles, this approximation being cared, following Born, by assigning them suitable "effective" charges. Moreover, still following Born, suitable repulsive forces are introduced. Finally, mutual electric forces are taken into account, in the approximation in which retardation is neglected. The long range order of the latter forces plays indeed a fundamental role, and compels one to deal with an actually infinitely extended system. Thus, in order to be able to deal (numerically) with a concretely finite number of particles (say of the order of 500 or 4000), one works with a model of a finite number of particles in a box, but with periodic boundary conditions. The long range forces are then estimated through the standard Ewald resummation procedure.

The only difference with respect to the quantum treatment is that the motions of the ions are governed by the Newton equations (with no Planck con- 
stant at all), rather than by the Schrödinger equation. So the Newton equations are solved by standard algorithms (as usually done in the so called Molecular Dynamics, or MD, simulations), exactly as for the FPU model. Having thus available at each time the positions of the ions, and so also the displacements from their equilibrium positions, the polarization $P(t)$ of the box is obtained in the standard elementary way (also averaging over several initial data). So, as is well known (see for example [15]), the electric susceptibility $\chi(\omega)$ is obtained, in the Green-Kubo frame, as the time Fourier transform of essentially the time autocorrelation of polarization. Then the dielectric function $\epsilon(\omega)$ is given as usual by

$$
\epsilon(\omega)=1+4 \pi \chi(\omega),
$$

and this eventually gives the refractive index $n(\omega)$ as

$$
n(\omega)=\sqrt{\epsilon(\omega)} .
$$

The results were published in [13], and the most relevant one is reported here in Fig. 1, which shows the real part of the refractive index $n(\omega)$ vs $\omega$ for $\operatorname{LiF}$ at a temperature of $300 \mathrm{~K}$. The circles and the diamonds are the experimental data, and the full line is our theoretical curve. The agreement with the experimental data is rather impressive, and is perhaps better than the one obtained (still by Russian scientists, see [16]) through perturbation methods of QM. To understand how can this occur in a classical computation involving no Planck's constant at all, is an interesting open problem.

\section{Conclusions}

So we have illustrated how the optical spectra of ionic crystals in the infrared are reproduced by classical methods. The interesting fact is that the occurring itself of a line spectrum turns out to be a physical counterpart of the qualitative property that the considered dynamical systems present regular (indeed, suitably regular) motions. Truly, this was shown to occur for realistic dynamical systems involving a number of particles of order 500 or 4000 , and not of the order $10^{23}$ of the Avogadro number. But this remark can be inverted. Indeed, as our numerical results agree with the experimental ones (obviously obtained from macroscopic samples involving numbers of particles of the order of the Avogadro number), one seems to be authorized to conclude that the results would not essentially change if the calculations were repeated with enormously larger numbers of particles. In other terms, for realistic models the results pertaining to the thermodynamic limit are apparently already attained with the mentioned numbers of particles (provided periodic conditions are introduced).

Coming back to the general mathematical problem discussed in this paper with respect to the scientific interests of Kolya, namely, the possible persistence of regular motions in nearly-integrable Hamiltonian dynamical systems in the thermodynamic limit, we believe that the results illustrated here give a nonnegligible support to the conjecture that the regular motions should in general 
persist in the thermodynamic limit, at least for physically relevant models. This however should be true only in the weak sense of the statistical mechanics approach, which avoids to control all individual initial data, and not in the strong classical sense considered by Kolya.

To our knowledge, the only case of physical interest in which a statistical approach was considered in dynamical systems theory is perhaps that related to the probability of capture into resonance. In the Russian school, this problem was particularly addressed by Anatoly Neishtadt (or Tolya) who, by the way, also was the first scientist who appreciated (giving useful comments) the paper [8], just after a preprint had been written down.

Strangely enough, Kolya instead always appeared to be somehow reluctant to even taking into consideration the idea of a statistical approach in the sense illustrated here, to say nothing about the idea of a possible deep implication for the foundations of physics. However we dare to be confident that, with the results illustrated here, he would lend us a smile of support, were he to still be among us.

\section{References}

[1] Nekhoroshev, N.N., An exponential estimate of the time of stability of nearly-integrable Hamiltonian systems, Russ. Math. Surv., 1977, Vol. 32, pp. 1-65.

[2] Benettin, G., Galgani, L. and Giorgilli, A., A proof of Nekhoroshev's theorem for the stability times in nearly integrable Hamiltonian systems, $\mathrm{Ce}$ lestial Mech., 1985, Vol. 37, pp. 1-25.

[3] Fermi, E, Pasta, J. and Ulam, S., Studies of nonlinear systems, in Fermi, E., Collected Papers, Roma: Accademia Nazionale dei Lincei, 1965, Vol.II, pp. 978-993.

[4] Bocchieri, Scotti, A., Bearzi, B. and Loinger, A., Anharmonic chain with LennardJones interaction, Phys. Rev. A, 1970, Vol. 2, pp. 2013-2019.

[5] Galgani, L. and Scotti, A., Planck-like distribution in classical nonlinear mechanics, Phys. Rev. Lett., 1972, Vol. 28, pp. 1173-1176.

[6] Cercignani, C., Galgani L. and Scotti A., Zero-point energy in classical nonlinear mechanics, Phys. Lett. A, 1972, Vol 38, pp.403-405.

[7] Izrailev, F.M. and Chirikov, B.V., Statistical properties of a nonlinear string, Sov. Phys. Dokl., 1966, Vol. 11, pp. 30-34.

[8] Carati, A., An averaging theorem for Hamiltonian dynamical systems in the thermodynamic limit, J. Stat. Phys., 2007, Vol. 128, pp. 1057-1077.

[9] Maiocchi, A., Bambusi, D. and Carati, A., An averaging theorem for FPU in the thermodynamic limit, J. Stat. Phys., 2014, Vol. 155, pp. 300-322. 
[10] Carati, A. and Maiocchi,A., Exponentially long stability times for a nonlinear lattice in the thermodynamic limit, Comm. Math. Phys., 2012, Vol 314, pp. 129-161.

[11] Giorgilli, A., Paleari, S. and Penati T., An extensive adiabatic invariant for the Klein-Gordon model in the thermodynamic limit, Annales Henri Poincare, 2015, Vol. 16, pp. 897-959.

[12] De Roeck, W. and Huveneers, F., Asymptotic localization of energy in nondisordered oscillator chains, Comm. Pure Appl. Math., 2015, Vol. 68, pp $1532-1568$.

[13] Gangemi, F., Carati, A., Galgani, L., Gangemi, R. and Maiocchi, A., Agreement of classical Kubo theory with the infrared dispersion curves $n(\omega)$ of ionic crystals, Europhys. Lett., 2015, Vol. 110, pp. 47003.

[14] Gangemi, F., Gangemi, R., Carati, A., Maiocchi, A. and Galgani, L, Infrared optical properties of $\alpha$-quartz by Molecular Dynamics simulations, Europhys. Lett., in print.

[15] Carati, A. and Galgani, L., Classical microscopic theory of dispersion, emission and absorption of light in dielectrics, Eur. Phys. J. D, 2014, Vol. 68, pp. 307.

[16] Ipatova, I.P., Maradudin, A.A. and Wallis, R.F. Temperature dependence of the width of the fundamental lattice-vibration absorption peak in ionic crystals. II. Approximate numerical results, Phys. Rev., 1967, Vol. 155, pp. 882-895. 